Journal of

(c) All rights are reserved by Butterworth RF.

\title{
Amantadine for the Treatment of Parkinson's Disease and its Associated Dyskinesias
}

\begin{abstract}
Keywords: Parkinson's disease; Amantadine; Dyskinesia; Nigrostriatal; Corticospinal; NMDA; RCT; Meta-analysis; Systematic review; Adverse events

\section{Abstract}

Disturbances of motor function characteristic of Parkinson's Disease (PD) are commonly treated with L-Dopa. However, prolonged treatment commonly results in L-Dopa-Induced Dyskinesias (LIDS) with high negative impact on patient's quality of life that seriously limits the use of L-Dopa. Amantadine, like L-Dopa, is effective for the replenishment of defective dopamine production in PD by mechanisms involving increased synthesis and decreased synaptic reuptake with consequent improvements of the patient's motor symptoms. Results of RCTs and meta-analyses continue to support the claim that amantadine is effective for the treatment of early or stable PD. Preclinical and clinical studies reveal that LIDs result from modifications of corticostriatal (glutamatergic) and nigrostrial (dopaminergic) connectivity resulting from the relative over-activation of NMDA receptors, a phenomenon shown to occur in patients with LIDs using Positron Emission Tomography. In addition to its beneficial actions in restoring dopaminergic function, amantadine is a potent non-competitive NMDA receptor antagonist and, as such, affords a potentially effective agent for the treatment for LIDs. Indeed, beneficia effects of amantadine for the treatment of LIDs have been described in multiple Randomized Controlled Trials (RCTs) using a range of wellestablished dyskinesia rating scales over the last two decades and extended-release formulations of amantadine have also been found to be effective. Confirmation of clinical efficacy of amantadine for the treatment of LIDs has been complemented by the results of systematic reviews and meta-analyses that include a Movement Disease Society (MDS)-commissioned evidence-based update of treatment options. Treatment of PD patients with amantadine during the COVID-19 pandemic could be advantageous since, in addition to its ability to correct the movement disorder and dyskinesias, amantadine has the potential to limit replication of SARS-CoV-2, the virus responsible for COVID-19.
\end{abstract}

\section{Introduction}

In 1968, a 58-year-old patient with moderately severe PD reported that, while taking amantadine to prevent symptoms of the flu, a remarkable remission of cogwheel rigidity and tremor was found to occur. The PD symptoms promptly reappeared upon cessation of amantadine. This serendipitous observation led to a trial in 1969 in 163 patients with PD two thirds of whom showed significant clinical benefit [1]. These results opened the way for decades of fundamental and clinical research into the mechanisms involved and evidence base for the efficacy of amantadine for the treatment of PD and for USFDA approval a short time thereafter.

From the molecular structural standpoint, amantadine has an adamantine backbone with an amino group substituted at one of the four methyne positions giving rise to the molecule 1-aminotricyclo [3.3.1.1.1,7] decane.
Parkinson's disease $\&$ Alzheimer's disease

\author{
Butterworth Roger F* \\ Department of Medicine, University of Montreal, Canada \\ *Address for Correspondence \\ Butterworth Roger F, Professor of Medicine, University \\ of Montreal, Montreal, Qc, Canada 45143 Cabot Trail, \\ Englishtown, NS, B0C 1H0, Canada; E-mail: rb@enceph.com \\ Submission: 1- July- 2020 \\ Accepted: 5- August- 2020 \\ Published: 7- August- 2020 \\ Copyright: (c) 2020 Butterworth RF. This is an open access article \\ distributed under the Creative Commons Attribution License, which \\ permits unrestricted use, distribution, and reproduction in any medium, \\ provided the original work is properly cited.
}

Mechanisms of action of amantadine in the treatment of the motor symptoms of PD

$\mathrm{PD}$ is an age-related neurodegenerative disease characterized by progressive degeneration of dopaminergic neurons. The neurodegenerative process in $\mathrm{PD}$ is characterized by a loss of dopamine-secreting cells in the substantia nigra. The most-widely employed treatment for PD is L-Dopa, the metabolic precursor for dopamine; the transformation from L-Dopa to dopamine is catalyzed by the enzyme L-Dopa Decarboxylase (DDC) located in the presynaptic nerve terminal (Figure 1). L-Dopa serves to replenish the precursor pool leading to increased dopamine synthesis.

Amantadine and the dopamine system: Amantadine, like L-Dopa, is also able to prevent the reduction of dopaminergic synaptic activity in PD and this, via multiple putative mechanisms. Studies in a range of in vitro synaptic preparations reveal that amantadine has the potential to increase dopamine synthesis, turnover and release and similar actions have been reported in vivo [2-5]. An additional proposed mechanism involves the direct inhibition of dopaminereuptake by amantadine that has been demonstrated in both in vitro preparations [6] and in vivo [3].

Anti-Parkinson activity of amantadine: Role of glutamate (NMDA) receptors: The potential for amantadine to increase dopamine synthesis from L-Dopa has been attributed, at least in part, to the drug's effect as an antagonist of NMDA receptors [7]. Support of the novel concept of a dynamic functional interaction between NMDA receptor activation and the release of dopamine in striatum was demonstrated directly in experimental animals using the technique of in vivo cerebral micro-dialysis in which perfusion of amantadine (0.1-1 mM) via the micro-dialysis probe resulted in increases in $\mathrm{Ca}^{2+}$ dependent release of dopamine. Moreover, iv administration of the NMDA receptor antagonist MK 801 significantly attenuated the amantadine-induced increase in striatal dopamine release [5]. These findings support the concept of an interaction between dopaminergic and glutamatergic transmission in the regulation of striatal dopamine release (Figure 1).

Translation of the hypothesis that stimulation of DDC activity 
Citation: Butterworth RF. Amantadine for the Treatment of Parkinson's Disease and its Associated Dyskinesias. J Parkinsons Dis Alzheimer Dis. 2020;7(1): 7 .

occurs as the result of NMDA receptor antagonism by amantadine to the human condition made use of the technique of Positron Emission Tomography (PET) and the ligand 6-[ $\left.{ }^{18} \mathrm{~F}\right]$-Fluoro-L-Dopa [7]. The study involved the measurement of radioactivity in brains of normal human volunteers following iv administration of the ${ }^{18} \mathrm{~F}$ PET ligand under baseline conditions and again following three consecutive days of treatment with amantadine $(100 \mathrm{mg} / \mathrm{d} \mathrm{po})$. Data from several brain regions of interest were obtained and coefficients of in situ ${ }^{18} \mathrm{~F}$-L-Dopa decarboxylation were calculated. Amantadine treatment resulted in significant $12 \%, 28 \%$ and $27 \%$ increases in caudate nucleus, putamen and ventral striatum respectively. These findings are consistent with stimulation of DDC activity in striatum of healthy human brain secondary to NMDA receptor antagonism by amantadine.

Other proposed mechanisms: Serotonin (5HT) neurons express the genetic and metabolic machinery necessary for dopamine synthesis and synaptic release. Chronoamperometric studies in rodent models of PD suggest a central role for the 5HT system in L-Dopa-derived dopamine synthesis and the potential for L-Dopainduced deterioration of $5 \mathrm{HT}$ function to reduce the clinical efficacy of L-Dopa and to promote motor side effects [8].

Additionally, based on studies in primary cultures of dopaminergic neurons, microglia and astroglia, it has been suggested that clinicallyrelevant concentrations of amantadine have the potential to manifest neuroprotective properties resulting from a dual mechanism of action involving a reduction in release of proinflammatory factors from activated microglia together with increased expression of the neurotrophic factor GDNF [9].

Evidence for the efficacy of amantadine for treatment of motor symptoms of $\mathrm{PD}$

Evidence from systematic reviews and meta-analyses: A Cochrane Review of RCTs [10] selected 6 trials that compared amantadine to placebo with or without L-Dopa or anticholinergic drugs. The studies included 215 patients treated for periods of from 6 to 64 weeks and amantadine doses of 100-200 mg/day. Exclusion criteria included patients with non-idiopathic forms of $\mathrm{PD}$, patients who had previously undergone stereotactic surgery as well as uncontrolled or non-randomized trials. Unfortunately, methodological limitations together with potential sources of bias meant that it was not possible to draw firm conclusions regarding the efficacy or safety of amantadine for the treatment of idiopathic PD.

On the other hand, the MDS published an evidence-based report of 7 studies on amantadine monotherapy and 14 studies on the efficacy of amantadine as an adjunct therapy to anticholinergics or L-Dopa concluded that amantadine was likely to be efficacious at controlling PD symptoms [11]. The MDS subsequently commissioned a review by way of update on treatments for the motor symptoms of PD that covered the findings from trials published in the period ending 31 December 2016 [12]. They concluded that amantadine was likely efficacious and clinically useful as symptomatic monotherapy as well as symptomatic adjunct therapy in early or stable PD. However, there was insufficient evidence for the effective treatment of motor fluctuations.

More recently and as part of a systematic review with meta- analysis of the efficacy of amantadine for the treatment of the motor symptoms of PD, electronic searches of Medline, PubMed, Cochrane Library and other databases up to May 2016 were performed using appropriate keywords yielding 9 placebo-controlled RCT's and 303 patients. Results demonstrated that amantadine was of significant benefit for improvement of PD motor symptoms as assessed by UPDRS III scores even at advanced stages of the disorder with [MD: $-0.29\{95 \%$ CI: $-0.53,-0.06\} Z=2.49, p=0.01]$. Details relating to the efficacy of independent trials are shown in the Forest Plot [13] (Figure 2).

Amantadine for the treatment of motor fluctuations in PD: The effect of amantadine on motor fluctuations was investigated in advanced PD [14]. Amantadine significantly lessened the severity of motor fluctuations; diary scores were lower with amantadine versus placebo $(\mathrm{n}=9$; mean, $1.03 \pm 0.12$ vs $1.62 \pm 0.16, \mathrm{p}<0.01$; variance, $1.3 \pm$

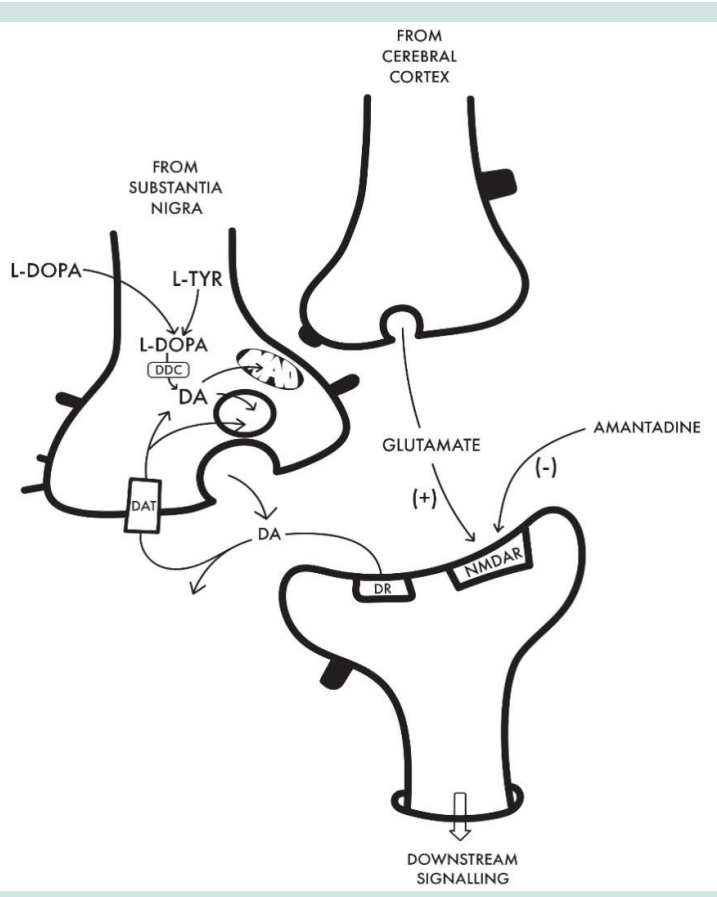

Figure 1: Schematic representation of cellular interactions implicated in the beneficial effects of L-Dopa and amantadine that underpin the beneficial effects of these agents for the treatment of the motor symptoms and dyskinesias associated with PD. On the left is a dopaminergic nerve terminal of a nigrostriatal fibre with key elements involved in dopamine (DA) synthesis via the two-step process from L-tyrosine (L-TYR) to L-DOPA via the enzyme tyrosine hydroxylase followed by the action of L-Dopa decarboxylase (DDC) to DA that is either stored in the synaptic vesicle prior to release into the synaptic cleft or converted to metabolites via monoamine oxidase (MAO). Released DA then activates its post-synaptic receptor (DR) or is reuptaken via the DA transporter (DAT).

Both L-Dopa and amantadine have the potential to replenish the nigrostriatal DA deficit characteristic of PD; L-Dopa by supplying the substrate for DDC and amantadine by stimulation of DA synthesis coupled with the blocking of DA re-uptake by mechanisms that have not been completely elucidated.

Benefit of amantadine for the treatment of L-Dopa-induced dyskinesias (LIDs) is generally attributed to the drug's properties as a non-competitive antagonist of the NMDA subclass of glutamate receptors (NMDAR). Blockade of these receptors results in the modulation of the interactions between corticostriatal glutamatergic and nigrostriatal dopaminergic inputs leading to improvements in LIDs. 
Citation: Butterworth RF. Amantadine for the Treatment of Parkinson's Disease and its Associated Dyskinesias. J Parkinsons Dis Alzheimer Dis. 2020;7(1): 7

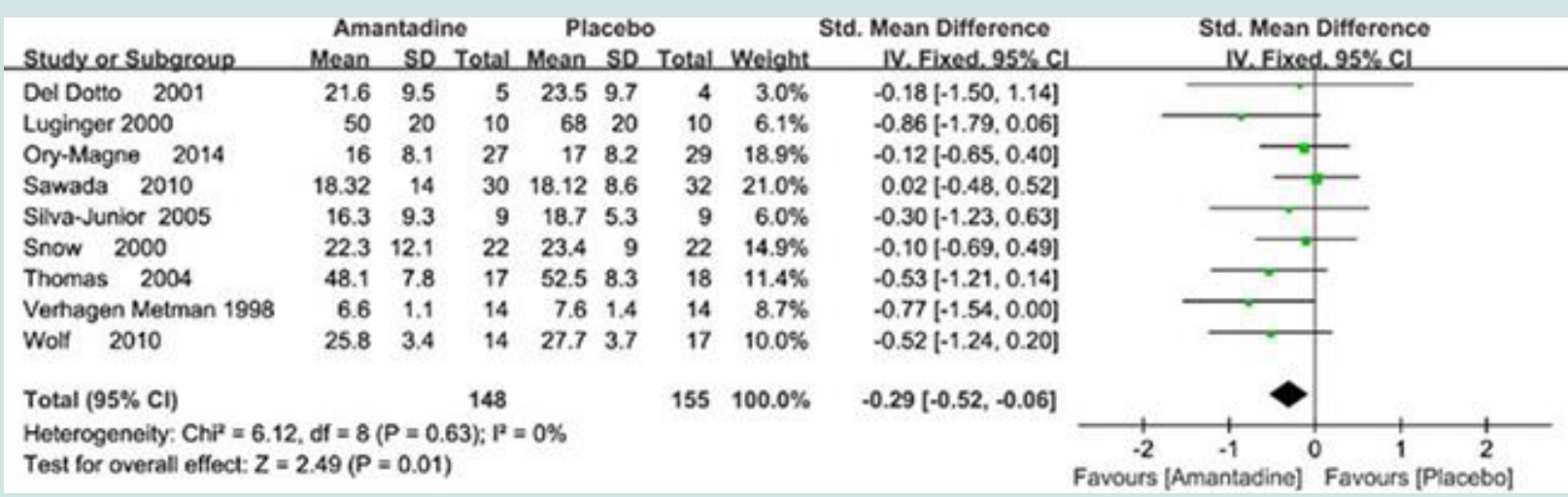

Figure 2: Forest plot of motor symptoms of PD assessed using UPDRS III scores [Mean \pm SD by Fixed Odds $\pm 95 \%$ Cl] following treatment with amantadine versus placebo. Individual trials are identified by 1st author's name and year. Full references are available in the reference list [13].

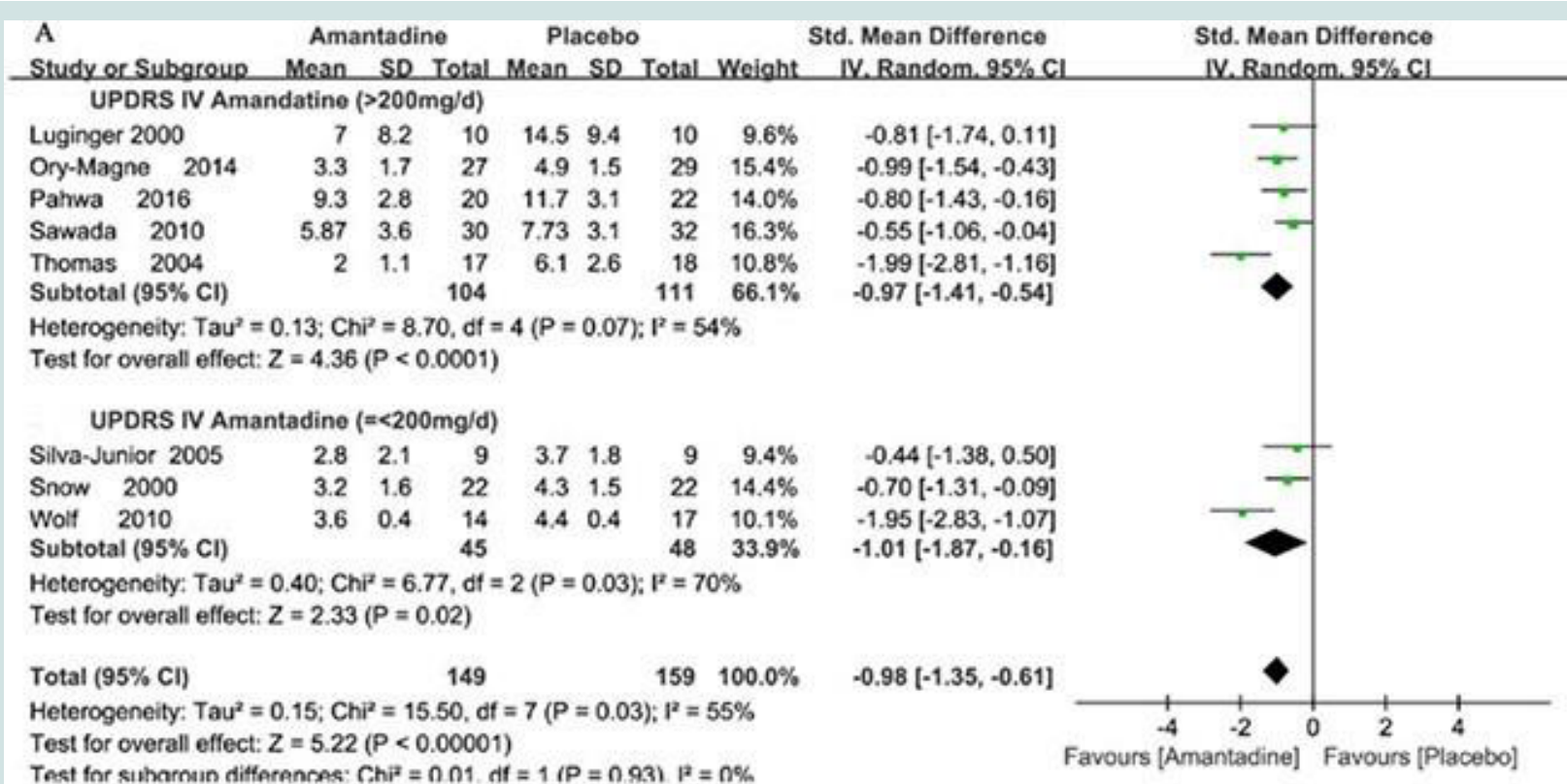

Figure 3: Forest plots of L-Dopa-induced dyskinesias in PD assessed using DRS scores [Mean \pm SD by Random Odds $\pm 95 \% \mathrm{Cl}$ ] following treatment with amantadine versus placebo as a function of the dose of amantadine [up to or greater than $200 \mathrm{mg} / \mathrm{d}$ ]. Data from 9 individual trials are identified by $1 \mathrm{st}$ author's name and year. Full references are available in the reference list [13]

0.3 vs $3.3 \pm 0.5, \mathrm{p}<0.01)$ and the duration of daily 'OFF' time declined while on amantadine ( $\mathrm{n}=14$; UPDRS IV item 39,1 [0-2] vs 1.5 [13]; $\mathrm{p}<0.001$ ). Activities of daily living while 'ON' and 'OFF' (UPDRS II) were also improved with amantadine. The effect of amantadine on motor fluctuations in PD patients was also investigated in an open-label infusion study [15]. Based on patients' diary notes, there was a reduction of $38 \%$ in the mean duration of 'OFF' times during amantadine infusion and improvement of $53 \%(\mathrm{p}<0.001)$.

Freezing of gait (FOG) is one of the most disturbing symptoms in advanced PD as well as in some atypical PD syndromes. FOG is often refractory to dopaminergic drugs [16]. There is evidence for the therapeutic use of amantadine for FOG in two RCTs [17, 18].

Akinetic crises such as acute akinesia, neuroleptic malignant and neuroleptic malignant-like syndrome, and Parkinsonism- hyperpyrexia syndrome can be successfully treated by acute administration of amantadine [19].

Amantadine has been reported to be of benefit in patients with progressive supranuclear palsy (PSP) [20], and in 14 patients with cortico-basal degeneration [21]. Reports on the open-label use of amantadine in multiple system atrophy (MSA) suggest variable antiparkinsonian efficacy [22].

\section{Amantadine for the Treatment of L-Dopa-Induced Dyskinesias (LIDs) in PD}

Dyskinesias occur in up to $90 \%$ of patients with PD treated with L-Dopa for ten years or more. Such dyskinesias manifest as increased spontaneous motricity in the form of hyperkinesias occurring at high plasma levels of L-Dopa and are also known as 'peak-dose dyskinesias'. Amantadine has been successfully employed in the 
Citation: Butterworth RF. Amantadine for the Treatment of Parkinson's Disease and its Associated Dyskinesias. J Parkinsons Dis Alzheimer Dis. 2020;7(1): 7

management of this type of LID.

Mechanisms of action of amantadine in the treatment of LIDs in PD

LIDs are generally considered to result from biochemical mechanisms in striatal neurons that result from rapibly-changing exposure to dopamine [23]. More recent investigations provide convincing evidence that these changes in the dopamine system are, in large part, the result of alterations in corticostriatal connectivity through changes in functional activity of NMDA receptors. Thus, as depicted in Figure 1, the corticostriatal (glutamatergic) and nigrostriatal (dopaminergic) input stimuli converge at the striatum where they have a key modulating influence on neuronal activity and consequently on motor control. Using the technique of PET and the ligand 11C-N-methyl-3-(thiomethylphenyl)-cyanamide, a marker of activated NMDA receptor channels, glutamatergic function in patients with PD with and without LIDs were compared [24]. Patients were assessed twice; after taking L-Dopa and again after withdrawal from it. Striatal uptake of tracer was calculated. Tracer uptake measured in the 'ON' condition following L-Dopa was higher in dyskinetic patients compared to patients without dyskinesia. These findings were consistent with those previously observed in animal studies and suggest that increased glutamatergic synaptic activity is implicated in the pathogenesis of LIDs. Amantadine is a noncompetitive antagonist of the NMDA receptor [25]. Consequently, blockade of NMDA receptors by amantadine has the potential to provide an approach to the control of LIDs in PD.

Efficacy of amantadine for the treatment of LIDs; results of systematic reviews and meta-analyses

Electronic searches of The Cochrane Controlled Trials Register issue 3 (2001), Medline (1966-2001), Embase (1974-2001), Clinicaltrials.gov (2001) and other databases as well as manual searches of reference lists from selected studies/reviews were examined in order to select RCTs comparing amantadine with placebo for the treatment of dyskinesias in patients with a clinical diagnosis of idiopathic PD. Three RCTs satisfied search criteria and were doubleblind crossover trials for a total of 53 patients. Regrettably, as a result of inadequate trial quality, missing data, lack of washout interval data, reviewers concluded that evidence was insufficient to determine safety or efficacy of amantadine for treatment of LIDs in patients with PD [10].

Searches of PubMed (1990-2010), Medline (1966-2010), Embase (1974-2010) and other databases with appropriate keywords were used resulting in 11 RCTs for a total of 253 PD patients with peakdose LIDs. The outcome measures were various dyskinesia rating scales and the Unified Parkinson's Disease Rating Scale (UPDRS) III and IV. The analysis showed a significant reduction in Standard Mean Difference (SMD) for UPDRS IV (SMD - 1.45; 95\% CI: - 2.28 to -0.63) and UPDRS III (SMD -0.41; 95\% CI: -0.69 to -0.12 ) after treatment with amantadine. Overall the meta-analysis confirmed the short-term benefits of amantadine therapy in the treatment of LIDs [26].

In the most recent MDS-commissioned evidence-based medicine review of treatments for the motor symptoms of PD covering the results of trials up to December 31, 2016, new conclusions for the treatment of dyskinesias were reported in which 3 new high-quality studies using amantadine were identified [12, 27-29]. In line with the conclusions from previous MDS recommendations, the use of amantadine for the treatment of dyskinesias in $\mathrm{PD}$ was judged to be both efficacious and clinically useful.

A contemporary up-dated meta-analysis of the efficacy of amantadine for the treatment of dyskinesias in PD made use of electronic searches of Medline, PubMed, Cochrane Library and other databases revealed a total of 11 eligible RCTs involving 356 patients with PD and LIDs. Making use of UPDRS IV and Dyskinesia Rating Scale (DRS) as measures of efficacy outcomes, highly significant benefits $(p<0.0001)$ were shown for amantadine [13]. Forest plots of dyskinesia assessment comparisons on UPDRS IV for amantadine versus placebo as a function of drug dosage [MD: - 0.98 \{95\%CI: - 1.35, $-0.61\} \mathrm{Z}=5.22, \mathrm{p}<0.00001]$ and trial design (parallel $v s$ crossover). Details are shown in Figure 3.

Efficacy of amantadine for the treatment of LIDs; results of individual RCTs

Benefits of oral or intravenous formulations of amantadine have been evaluated in patients with PD and LIDs in 10 RCTs the key elements of which are summarized as follows:

Sawada et al. [2010]: In a 27-day trial carried out in 36 patients with PD and LIDs, amantadine treatment $(300 \mathrm{mg} / \mathrm{d})$ was associated with a significant improvement of $64 \%$ in the Rush Dyskinesia Rating Scale versus $16 \%$ of placebo patients. The adjusted odds ratio for improvement by amantadine was 6.7 (95\% Confidence Interval [CI], 1.4-31.5, p<0.016); UPDRS IVa, dyskinesia improved to an even greater degree following amantadine treatment [27].

Wolf et al. [2010]: The long-term anti-dyskinetic effect of amantadine was evaluated in 32 patients with PD who were receiving L-Dopa and who had been on stable amantadine therapy for at least 1 year [30]. In patients who had been switched to placebo at the 3-week follow-up, there was a significant increase in dyskinesia, as measured with UPDRS item 32-33, but there was no significant change between baseline and follow-up in patients who had continued on amantadine.

Da Silva Junior et al. [2005]: The effects of amantadine on LIDs were assessed in 18 consecutive PD patients in an RCT [31]. The primary outcomes were improvements in CDRS and UPDRS IVa scores. Secondary outcomes were improvements in UPDRS II and III scores. Amantadine did not change the CDRS score for hyperkinesia or dystonia but decreased the duration of LIDs and its influence on daily activities $(\mathrm{p}=0.04)$ and UPDRS II score $(\mathrm{p}=0.01)$ more than placebo. These findings show that amantadine reduces the duration of LIDs and improves motor disability in PD.

Thomas et al. [2004]: A trial of amantadine was conducted in 14 PD patients patients treated with L-Dopa for $7.5 \pm 2.2 \mathrm{yrs}$ with motor fluctuations and dyskinesias evaluated by UPDRS IV and blinded videotape-based ratings using DRS. After 15 days of amantadine treatment, total dyskinesia scores decreased by $45 \%$, and there was a reduction in UPDRS IV item 32-34 scores compared with baseline and placebo $(\mathrm{p}<0.001)$. The mean positive duration of the effect in reducing dyskinesias was 4.9 months for amantadine versus 1.3 months for placebo $(\mathrm{p}<0.001)$, [32].

Del Dotto et al. [2001]: Nine patients with PD and severe peak- 
Citation: Butterworth RF. Amantadine for the Treatment of Parkinson's Disease and its Associated Dyskinesias. J Parkinsons Dis Alzheimer Dis. 2020;7(1): 7

dose dyskinesias received L-DOPA followed by amantadine (200 mg iv) or placebo and were assessed by UPDRS, motor exam \& AIMS. Amantadine improved dyskinesias by $50 \%$ with no loss of benefit for the motor symptoms of PD [33].

Luginger et al. [2000]: A 5-week, crossover trial examined the effect of amantadine on LIDs. Ten of 11 patients completed the study. Dyskinesia severity, following oral L-Dopa challenges, was significantly reduced by $52 \%$ after amantadine treatment (baseline score $14.5 \pm 9.4 v s$ post-treatment score $7.0 \pm 8.2 ; \mathrm{p}<0.05)$ but not placebo (baseline score $16.6 \pm 11.4$ vs post-placebo score $15.5 \pm 12.1$; $\mathrm{p}>0.05)$. Similarly, amantadine was associated with a significant reduction of $53 \%$ in the cumulative dyskinesia score in self-scoring dyskinesia diaries (post-amantadine treatment period $11.9 \pm 11.4 \mathrm{vs}$ post-placebo period $25.6 \pm 16.7 ; \mathrm{p}<0.05$ ). Dyskinesia duration and dyskinesia disability, as measured by UPDRS IV items 32-33, were also significantly reduced by amantadine (baseline score $3.4 \pm 0.3 \mathrm{vs}$ post-treatment score $1.7 \pm 0.5$; $\mathrm{p}<0.05)$ [34].

Snow et al. [2000]: A trial examined the effect of amantadine on LIDs in 22 patients with PD. Patients were treated with amantadine (up to $200 \mathrm{mg} / \mathrm{d}$ for 3 weeks) in addition to other antiparkinsonian medications. Dyskinesias were evaluated following an oral L-Dopa challenge. Amantadine treatment significantly reduced the total dyskinesia score by $24 \%$; this was also reflected in the subjects perception that dyskinesias, measured by UPDRS III and IV, were reduced [35].

Verhagen Metman et al. [1998]: A trial evaluated amantadine for treatment of dyskinesias in 18 patients with advanced PD. Patients, who had already been treated with L-Dopa for a mean period of 12 years, were given amantadine (up to $400 \mathrm{mg}$ /day) or placebo for 3 weeks. Signs and symptoms of PD and dyskinesias were recorded at the end of the 3-week treatment period during intravenous L-Dopa infusions over $7 \mathrm{~h}$ under steady-state conditions. Amantadine reduced dyskinesia severity by $60 \%(\mathrm{p}=0.0001)$ compared to placebo in completers and this was not accompanied by aggravation of the signs and symptoms of PD [36]. Beneficial effects of amantadine on motor response complications were maintained in a 1-year follow-up study in which a $56 \%$ reduction in dyskinesia was noted with amantadine versus $60 \%$ in the initial study. Motor symptoms associated with L-Dopa were still improved [14].

Goetz et al. [2013]: Assessment of the effects of treatment of patients with $\mathrm{PD}$ and dyskinesia with amantadine (up to $300 \mathrm{mg} / \mathrm{d}$ for 8 weeks) versus placebo were made by comparing the sensitivity to treatment effects at 4 and 8 weeks using 8 different dyskinesia rating scales. Four of the eight scales (UDysR, Lang-Fahn, PDys-26 and CGI-C) demonstrated a significant improvement in dyskinesia after 8 weeks treatment with amantadine $v s$ placebo [28].

Ory-Magne et al. [2014]: The AMANDYSK trial was a 3-month, multicenter, parallel group/wash out in single line RCT in patients with PD. Fifty-seven dyskinetic patients who had received amantadine at a dosage of $200 \mathrm{mg} / \mathrm{d}$ for $\geq 6$ months were included in the study. Washout of oral amantadine significantly worsened LIDs where UPDRS IV item 32-33 deteriorated more in patients who switched to placebo ('discontinuing' group; $+1.7 \pm 2.0$ units; 95\% CI: 0.9-2.4) compared with those maintained on amantadine ('continuing' group; $+0.2 \pm 1.5$ units; $95 \%$ CI: -0.4 to $0.8 ; \mathrm{p}=0.003$ ). Patients in the discontinuing group also had more dropouts for LIDs, a greater increase in 'ON' time with troublesome dyskinesia, and a greater worsening of Abnormal Involuntary Movement Scale scores. No significant effect of amantadine on PD motor symptoms was observed [29].

Efficacy of amantadine (extended release) for the treatment of LIDs: results of RCTs and a meta-analysis

The last 5 years have witnessed the publication of two RCT's and a meta-analysis in which the efficacy of amantadine (extended-release formulation) for the treatment of LIDs were described. A report of the efficacy of ADS-5102, a long-acting, extended -release formulation of amantadine has appeared [37]. In a parallel group phase 2/3 RCT 83 PD patients with LIDs were assigned to one of three doses of ADS5102 or placebo for an 8-week period making use of UDysRS, UPDRS and other instruments. The drug was well tolerated and resulted in significant and dose-dependent improvements in dyskinesia. A follow-up phase 3 trial of the same agent essentially confirmed the above results showing significant benefit for both dyskinesias and OFF times [38].

To determine the effectiveness and safety of ADS-5102 for LIDs in patients with $\mathrm{PD}$, a meta-analysis of relevant trials was undertaken. Electronic databases were searched on or before March 1, 2019 for relevant trials. Only RCTs using ADS-5102 for LID in PD were included. ADS-5102 led to a reduction in dyskinesia scores (mean difference: -9.56 : CI: -10.05 to $-9.07 ; \mathrm{p}<0.00001)$ and 'ON' times without troublesome dyskinesia (mean difference 2.50: CI 2.38 to 2.63; $\mathrm{p}<0.00001)$ [39].

\section{Amantadine treatment of PD during the COVID-19 pandemic}

PD and COVID-19 share multiple common features including age-dependency of disease severity and an association with comorbidities such as diabetes, respiratory problems and cardiovascular disorders. Concerns have been raised regarding the potential effects of COVID-19 on PD severity and, conversely, on the effects of PD on immune status that could impact disease outcome in COVID-19. Symptoms of PD are known to deteriorate during systemic infections and diagnostic features of COVID-19 such as fever, fatigue and stress are known to not only aggravate tremor, gait disturbances and dyskinesias in PD but may also compromise the efficacy of L-Dopa [40].

Concerns on the likelihood of interactions between COVID-19 and PD with the potential to result in the clinical worsening of both conditions described above may be mitigated once treatment with amantadine is initiated. Recent investigations provide convincing evidence for significant benefit of amantadine for the treatment of COVID-19 per se. Two independent mechanisms have been proposed namely 1 . The down-regulation of expression of host-cell proteases leading to impaired release of the viral genome into the host-cell cytoplasm and 2. Activation of NMDA receptors has been implicated in the pathogenesis of the acute respiratory failure characteristic of COVID-19 [41]. The pertinence of these mechanisms of action of amantadine in relation to the management of COVID-19 in patients with $\mathrm{PD}$ is worthy of further investigations in future research. 
Citation: Butterworth RF. Amantadine for the Treatment of Parkinson's Disease and its Associated Dyskinesias. J Parkinsons Dis Alzheimer Dis. 2020;7(1): 7

\section{Adverse Events (AEs) associated with amantadine treatment for LIDs in PD}

AEs listed in the present review are largely taken from data provided in the reports of meta-analyses, systematic reviews and individual RCTs described in the text and relate to AEs using therapeutically-relevant concentrations of amantadine. Common AEs included visual hallucinations, confusion, blurred vision, foot edema and constipation. Behavioral symptoms characteristic of impulse control disorders including pathological gambling, hyper sexuality and compulsive spending/eating have been described. Other AEs include live do reticular is, nausea, dry mouth, dizziness and somnolence, peripheral edema, anxiety and depression $[13,29,30,35,36]$. Similar AEs for the extended-release formulations of amantadine in the treatment of LIDs in PD have been reported $[37,38]$.

\section{Conclusions}

Amantadine is effective for the replenishment of defective synaptic DA turnover by mechanisms involving both increased synthesis and decreased release of the transmitter with consequent improvements of motor control in PD patients. LIDs in such patients, on the other hand, appear to be the consequence of modifications of both corticostriatal (glutamatergic) and nigrostriatal (dopaminergic) neurotransmitter systems leading to impaired striatal connectivity resulting from the relative over-activation of the NMDA subclass of glutamate receptors. Being an effective non-competitive NMDA antagonist, amantadine affords a potentially-effective agent for the treatment of LIDs. The functional interplay between glutamatergic and dopaminergic systems in striatum and beneficial effects of amantadine have been demonstrated using PET and the ligand 6- $\left[\mathrm{F}^{18}\right]$-Fluoro-L-Dopa in human brain.

Evidence in support of the efficacy of amantadine for the treatment of PD and its associated dyskinesias has been forthcoming in the form of numerous RCTs, systematic reviews and meta-analyses. The MDS published an updated evidence-based report of clinical trials in which amantadine monotherapy was effective for the control of the motor symptoms of PD and, more recently, a systematic review with meta-analysis confirmed that amantadine was of significant benefit for improvement of motor symptoms assessed by UPDRS even at advanced stages of the disorder. There is evidence to support the notion that amantadine may also be beneficial for the control of motor fluctuations in PD such as ON and OFF times as well as FOG and acute akinesia. Further high quality RCTs with systematic review and meta-analysis are necessary in order to confirm these reports.

Evidence for the efficacy of amantadine for the treatment of LIDs in patients with PD is provided by the results of 10 good-to-excellent quality RCTs published in the last two decades using a range of doses of amantadine from $100-300 \mathrm{mg} / \mathrm{d}$, a variety of well-established dyskinesia rating scales, and trial design (parallel, crossover) for treatment periods of up to 1 year. Publication of the successful results of RCTs and a meta-analysis of the efficacy of an extended-release formulation of amantadine for the treatment of LIDs will provide a choice of treatment options; trials comparing this formulation with the classic immediate-release formulation with flexible increasing $(100-400 \mathrm{mg} / \mathrm{d})$ doses of amantadine together with assessments of cost-effectiveness are now required. It appears that both immediaterelease and extended-release formulations of amantadine have associated AEs that include foot edema, blurred vision, visual hallucinations and constipation.

Amantadine treatment for PD patients has the potential to provide a treatment of choice during the COVID-19 pandemic given the growing body of evidence that, in addition to the well-established actions on motor performance and dyskinesias summarized in the present review, amantadine may also limit replication of the SARSCoV-2 virus that is responsible for COVID-19. This issue warrants further investigation at pace.

\section{References}

1. Schwab RS, England AC, Poskanzer DC, Young RR (1969) Amantadine in the Treatment of Parkinson's Disease. JAMA 208: 1168-1170.

2. Hesselink MB, De Boer AG, Breimer DD, Danysz W (1999) Dopamine release in the prefrontal cortex in response to memantine following subchronic NMDA receptor blockade with memantine: a microdialysis study in rats. J Neural Transm (Vienna) 106: 803-818.

3. Mizoguchi K, Yokoo H, Yoshida M, Tanaka T, Tanaka M (1994) Amantadine increases the extracellular dopamine levels in the striatum by re-uptake inhibition and by N-methyl-D-aspartate antagonism. Brain Res 662: 255-258.

4. Quack G, Hesselink M, Danysz W, Spanagel R (1995) Microdialysis studies with amantadine and memantine on pharmacokinetics and effects on dopamine turnover. J Neural Transm Suppl 46: 97-105.

5. Takahashi T, Yamashita H, Zhang YX, Nakamura S (1996) Inhibitory effect of MK-801 on amantadine-induced dopamine release in the rat striatum. Brain Res Bull 41: 363-367.

6. Heikkila RE, Cohen G (1972) Evaluation of amantadine as a releasing agent or uptake blocker for $\mathrm{H} 3$-dopamine in rat brain slices. Eur J Pharmaco I20: 156-160.

7. Deep P, Dagher A, Sadikot A, Gjedde A, Cumming P (1999) Stimulation of dopa decarboxylase activity in striatum of healthy human brain secondary to NMDA receptor antagonism with a low dose of amantadine. Synapse 34: 313-318.

8. Nevalainen N, Af Bjerkén S, Gerhardt GA, Strömberg I (2014) Serotonergic nerve fibers in L-DOPA-derived dopamine release and dyskinesia. Neuroscience 260: 73-86.

9. Ossola B, Schendzielorz N, Chen SH, Bird GS, Tuominenet RK, et al. (2011) Amantadine protects dopamine neurons by a dual action: reducing activation of microglia and inducing expression of GDNF in astroglia. Neuropharmacology 61: 574-582.

10. Crosby N, Deane KH, Clarke CE (2003) Amantadine in Parkinson's disease. Cochrane Database Syst Rev CD003468.

11. Lang AE, Lees A (2002) Management of Parkinson's disease: an evidencebased review. Mov Disord 4: 1-166.

12. Fox SH, Katzenschlager R, Lim SY, Barton B, de Bie RMA, et al. (2018) International Parkinson and movement disorder society evidencebased medicine review: Update on treatments for the motor symptoms of Parkinson's, disease. Mov Disord 33: 1248-1266.

13. Kong M, Ba M, Ren C, Yu L, Dong S, et al. (2017) An updated meta-analysis of amantadine for treating dyskinesia in Parkinson's disease. Oncotarget 8: 57316-57326.

14. Metman LV, Del Dotto P, LePoole K, Konitsiotis S, Fang J, et al (1999). Amantadine for levodopa-induced dyskinesias: a 1-year follow-up study. Arch Neurol 56: 1383-1386.

15. Růzicka E, Streitová H, Jech R, Kanvosky P, Roth J, et al. (2000) Amantadine infusion in treatment of motor fluctuations and dyskinesias in Parkinson's disease. J Neural Transm (Vienna) 107: 1297-1306. 
Citation: Butterworth RF. Amantadine for the Treatment of Parkinson's Disease and its Associated Dyskinesias. J Parkinsons Dis Alzheimer Dis. 2020;7(1): 7 .

16. Giladi N (2008) Medical treatment of freezing of gait. Mov Disord 23: 482-488.

17. Kim YE, Yun JY, Yang HJ, Kim HJ, Namyi Gu, et al. (2012) Intravenous amantadine for freezing of gait resistant to dopaminergic therapy: a randomized, double-blind, placebo-controlled, cross-over clinical trial. PLoS One 7: e48890.

18. Lee JY, Oh S, Kim JM, Kim JS, Eungseok Oh, et al. (2013) Intravenous amantadine on freezing of gait in Parkinson's disease: a randomized controlled trial. J Neurol 260: 3030-3038.

19. Thomas A, Onofrj M (2005) Akinetic crisis, acute akinesia, neuroleptic malignant-like syndrome, Parkinsonism-hyperpyrexia syndrome, and malignant syndrome are the same entity and are often independent of treatment withdrawal. Mov Disord 20: 1671-1672.

20. Kompoliti K, Goetz CG, Litvan I, Jellinger K, Verny M (1998) Pharmacological therapy in progressive supranuclear palsy. Arch Neurol 55: 1099-1102.

21. Kompoliti K, Goetz CG, Boeve BF, Maraganore DM, Ahlskoget JE, et al. (1998) Clinical presentation and pharmacological therapy in corticobasal degeneration. Arch Neurol 55: 957-961.

22. Wenning GK, Ben Shlomo Y, Magalhães M, Daniel SE, Quinn NP (1994) Clinical features and natural history of multiple system atrophy: An analysis of 100 cases. Brain 117: 835-845.

23. Jenner $P$ (2008) Molecular mechanisms of L-DOPA-induced dyskinesia. Nat Rev Neurosci 9: 665-677.

24. Ahmed I, Bose SK, Pavese N, Ramlackhansingh A, Turkheimeret F, et al (2011) Glutamate NMDA receptor dysregulation in Parkinson's disease with dyskinesias. Brain 134: 979-986.

25. Parsons CG, Panchenko VA, Pinchenko VO, Tsyndrenko AY, Krishtal OA (1996) Comparative patch-clamp studies with freshly dissociated rat hippocampal and striatal neurons on the NMDA receptor antagonistic effects of amantadine and memantine. Eur J Neurosci 8: 446-454.

26. Elahi B, Phielipp N, Chen R (2012) N-Methyl-D-Aspartate antagonists in levodopa induced dyskinesia: a meta-analysis. Can J Neurol Sci 39: 465-472.

27. Sawada H, Oeda T, Kuno S, Nomoto M, Yamamoto K, et al. (2010) Amantadine for dyskinesias in Parkinson's disease: a randomized controlled trial. PLoS One 5: e15298.

28. Goetz CG, Stebbins GT, Chung KA, Hauser RA, Miyasaki JM, et al. (2013) Which dyskinesia scale best detects treatment response? Mov Disord 28: 341-346.

29. Ory-Magne F, Corvol JC, Azulay JP, Bonnet AM, Brefel-Courbon C, et al. (2014) Withdrawing amantadine in dyskinetic patients with Parkinson's disease: the AMANDYSK trial. Neurology 82: 300-307.

30. Wolf E, Seppi K, Katzenschlager R, Hochschorner G, Ransmayr G, et al. (2010) Long-term antidyskinetic efficacy of amantadine in Parkinson's disease. Mov Disord 25: 1357-1363.

31. da Silva-Júnior FP, Braga-Neto P, Sueli Monte F, de Bruin VM (2005) Amantadine reduces the duration of levodopa-induced dyskinesia: a randomized, double-blind, placebo-controlled study. Parkinsonism Relat Disord 11: 449-452.

32. Thomas A, lacono D, Luciano AL, Armellino K, Di I, et al. (2004) Duration of amantadine benefit on dyskinesia of severe Parkinson's disease. J Neurol Neurosurg Psychiatry 75: 141-143.

33. Del Dotto P, Pavese N, Gambaccini G, Bernardini S, Metman LV, et al. (2001) Intravenous amantadine improves levadopa-induced dyskinesias: an acute double-blind placebo-controlled study. Mov Disord 16: 515-520.

34. Luginger E, Wenning GK, Bösch S, Poewe W (2000) Beneficial effects of amantadine on L-dopa-induced dyskinesias in Parkinson's disease. Mov Disord 15: 873-878.

35. Snow BJ, Macdonald L, Mcauley D, Wallis W (2000) The effect of amantadine on levodopa-induced dyskinesias in Parkinson's disease: a double-blind, placebo-controlled study. Clin Neuropharmacol 23: 82-85.

36. Verhagen Metman L, Del Dotto P, van den Munckhof P, Fang J, Mouradian MM, et al. (1998) Amantadine as treatment for dyskinesias and motor fluctuations in Parkinson's disease. Neurology 50: 1323-1326.

37. Pahwa R, Tanner CM, Hauser RA, Sethi K, Isaacson S, et al. (2015) Amantadine extended release for levodopa-induced dyskinesias in Parkinson's disease (EASED Study). Mov Disord 30: 788-795.

38. Oertel W, Eggert K, Pahwa R, Tanner CM Hauser RA, et al. (2017) Randomized, placebo-controlled trial of ADS-5102 (amantadine) extendedrelease capsules for levodopa-induced dyskinesia in Parkinson's disease (EASE LID ${ }_{3}$ ). Mov Disord 32: 1701-1709.

39. Pajo AT, Espiritu Al, Jamora RDG (2019) Efficacy and safety of extendedrelease amantadine in levodopa-induced dyskinesias: a meta-analysis. Neurodegener Dis Manag 9: 205-215.

40. Butterworth RF (2020) Amantadine, Parkinson's Disease and COVID-19. Covid perspect res \& rev 1: $1-6$.

41. Butterworth RF (2020) Amantadine treatment for Parkinson's Disease during COVID-19: Bimodal action targeting viral replication and the NMDA receptor. J Parkinsons Dis Alzheimer Dis 7: 1-4.

\section{Acknowledgement}

Research from the author's Unit including work on Parkinsonism and costs of publication of original articles and reviews was funded in part over the last two decades by The Canadian Institutes of Health Research (CIHR) and The Canadian Association for Study of The Liver (CASL). The author is grateful to $\mathrm{Mr}$ Jonas Eric Pilling for the design of Figure 1. 